\title{
Long-term individual marking of small freshwater fish: the utility of Visual Implant Elastomer tags
}

\author{
Arne Jungwirth $^{1,2}$ (D) Valentina Balzarini ${ }^{1,3} \cdot$ Markus Zöttl $^{1,4} \cdot$ Andrea Salzmann $^{2,1} \cdot$ Michael Taborsky $^{1}$. \\ Joachim G. Frommen ${ }^{1}$
}

Received: 23 November 2018 / Revised: 5 March 2019 / Accepted: 11 March 2019/Published online: 26 March 2019

(C) The Author(s) 2019

\begin{abstract}
Tracking wild animals over long periods of time is a non-trivial challenge. This has caused a bias in the availability of individualbased long-term datasets with the majority including birds and mammals. Visual Implant Elastomer (VIE) tags are now a widely used technique that may facilitate the collection of such data for fish and amphibians. However, VIE tags might have important drawbacks. Overall, four potential issues with VIE tags have been proposed: tag loss or misidentification, limited number of individual identifiers, enhanced mortality risk, and effects on intra-specific interactions. Here, we present three experiments in which we investigated these potential problems with VIE tagging in small freshwater fish both in the laboratory and in the wild, using the cooperatively breeding Lake Tanganyika cichlid Neolamprologus pulcher. We find VIE tags to be generally suitable for work with these fish as they did not impair survival, were recognisable up to 2 years after injection, and did not generally disturb group formation. Nevertheless, we identify specific issues of VIE tagging, including colour- and position-dependent variation in tag identification rates, and indications that specific colours may influence social behaviour. Our results demonstrate the suitability of VIE tags for long-term studies on small freshwater fish, while also highlighting the need of validating this method carefully for any species and study.
\end{abstract}

\section{Significance statement}

Information on the survival, dispersal, and reproductive success of wild individuals across their lifespan is among the most valuable data in Behavioural Ecology. Because tracking of free-ranging individuals over extended periods of time is challenging, there exists a bias in the taxonomic distribution of such long-term datasets. Here, we investigate the suitability of visible implant elastomers (VIE) as a tracking technique to allow for the collection of such data also in small tropical freshwater fish. We show that VIE tags neither alter social behaviour in our study species, nor do they reduce survival, but they enable the tracking of wild

Communicated by L. Z. Garamszegi

Electronic supplementary material The online version of this article (https://doi.org/10.1007/s00265-019-2659-y) contains supplementary material, which is available to authorized users.

Arne Jungwirth

arne.jungwirth@gmx.net

1 Division of Behavioural Ecology, Institute for Ecology and Evolution, University of Bern, Wohlenstrasse 50a, Hinterkappelen, 3032 Bern, Switzerland

2 Department of Zoology, University of Cambridge, Downing Street, Cambridge CB2 3EJ, UK

3 Centre for Ecology and Conservation, University of Exeter, Penryn Campus, Penryn, Cornwall TR10 9FE, UK

4 Ecology and Evolution in Microbial Model Systems, EEMiS, Department of Biology and Environmental Science, Linnaeus University, SE-391 82 Kalmar, Sweden individuals across years. We also identify colours and tag positions that are less suitable. We conclude that VIE tags can help produce long-term datasets also for small fish, provided certain precautions are met.

Keywords Elastomer tagging · VIE tags · Cichlid fish · Social behaviour $\cdot$ Survival $\cdot$ Individual identification

\section{Introduction}

Addressing many of the long-standing puzzles in behavioural and evolutionary biology requires high-quality, long-term, individual-based data from the wild (Clutton-Brock and Sheldon 2010). Progress towards long-standing questions like the eco-evolutionary background of ageing, or the evolutionary mechanisms underlying sociality and cooperation strongly depends on individual-based data gathered in long-term field 
studies (Nussey et al. 2013; Koenig and Dickinson 2016). The data produced by such work allow unravelling the links between genotype, phenotype, and the environment, ultimately providing insight into how and why evolution has afforded a given biological phenomenon (Clutton-Brock and Sheldon 2010). It would thus seem beneficial if more research programs were tracking free-ranging individuals over extended periods of time.

Long-term data from the wild are notoriously difficult to acquire and their collection suffers from at least two major limitations: first, devices for marking or tracking can have a considerable impact on those individuals bearing them (Murray and Fuller 2000). It must thus be assured that their effects are minimised and monitored. Second, variation exists in how well individuals of different species can be tracked. It is typically easier to follow larger animals, terrestrial species are usually more easily monitored than aquatic ones, and species with high levels of site-fidelity or small home-ranges are easier to track (Murray and Fuller 2000).

These limitations have led to biases with regard to the taxa for which information is available: most high-quality, long-term, individual-based data of freely roaming animals come from birds and terrestrial mammals. For example, much of our understanding of cooperative breeding in vertebrates is guided by work on these phylogenetic groups (Koenig and Dickinson 2016). Similarly, the majority of long-term studies of ageing in the wild focuses on placental mammals and birds (Nussey et al. 2013). While this research is highly valuable, it is not necessarily clear how far findings from these studies can be extrapolated to other taxonomic groups, e.g. amphibians, fishes, or invertebrates. An increase in the number of long-term studies tracking wild individuals of non-mammalian and non-avian taxa thus seems desirable. However, three of the most commonly used techniques to track birds and mammals (i.e. colour rings, radio collars, and passive integrated transponder (PIT) tags) do not work well for species that are small, have an exoskeleton, lack extremities that allow ringing, and/or are aquatic (Murray and Fuller 2000). Consequently, alternative techniques are required for such species. In the last decades, visible implant elastomer (VIE) tags have emerged as a viable method for marking animals. For VIE tagging, a silicone-based coloured liquid is injected subcutaneously. The liquid eventually solidifies and is thus resistant to biodegradation and consequently allows for long-term recognition of tags (North West Marine Technology 2008).

VIE tags have been used successfully in various species of vertebrates and invertebrates (e.g. blow flies: Moffatt 2013; lobsters: Neenan et al. 2015; fishes: Kozłowski et al. 2017; frogs: Sapsford et al. 2015; turtles: Anderson et al. 2015). Four main drawbacks of VIE tags have been reported. First, individuals may be misidentified because tags were (partially) lost, moved, or were misidentified, especially where times between tag injection and attempted identification are long (FitzGerald et al. 2004; Sapsford et al. 2015). Second, compared to other methods of marking, VIE tags offer a limited number of individual identifiers. How many unique marks can be applied depends on the number of different positions at which tags are implanted, the number of colours used, and the maximum number of tags that are placed on each individual (North West Marine Technology 2008). Third, tags may reduce survival of marked individuals, especially when bright colours are used on otherwise cryptic species (Catalano et al. 2001), when tagging affects the immune system (Henrich et al. 2014), or where particularly small individuals are tagged (Peterson et al. 2018). Fourth, intra-specific interactions may be influenced by the presence of VIE tags, e.g. when tags resemble parasite infections or when increased colourfulness raises attractiveness to potential mates (Frommen et al. 2015; Schuett et al. 2017). Consequently, the viability of VIE tagging for individual-based data collection of a given species should be tested in a broad range of contexts prior to largescale applications of tags. To date, most studies have focussed either on the impact of VIE tags on individual survival (e.g. Claverie and Smith 2007; Coombs and Wilson 2008; Neenan et al. 2015; Kozłowski et al. 2017), or, to a lesser extent, on behavioural changes caused by tags (e.g. Croft et al. 2004; Frommen et al. 2015; Schuett et al. 2017). Studies combining long-term field and laboratory data on tag retention, individual survival, and behavioural changes are scarce (but see Malone et al. 1999; Roberts and Kilpatrick 2004 for notable exceptions).

Here, we aim to scrutinise the suitability of VIE tagging for tracking a small tropical freshwater fish, the cichlid Neolamprologus pulcher. This species is endemic to Lake Tanganyika and has emerged as a suitable model for the study of sociality and cooperation (Wong and Balshine 2011; Taborsky 2016). We first investigate whether VIE tags influence social dynamics in these fish, as intra-specific individual recognition and social interactions appear to be strongly influenced by visual cues (Balshine-Earn and Lotem 1998; Kohda et al. 2015; Balzarini et al. 2017). Further, we study whether VIE tags can be used in nature to conduct individual-based, long-term surveys of these fish, and how such work might be influenced by the four caveats of VIE tagging outlined above. Our focus is especially on tag identification and on individual survival. We thus conducted three experiments, each focusing on a different aspect of VIE tagging in these fish: (i) in a "social integration experiment", we investigated whether VIE tags influence group formation, (ii) in a long-term laboratory experiment, we checked how well VIE tags can be identified under controlled laboratory conditions and how they influence individual survival in this context, and (iii) in a long-term field experiment, we studied VIE tag identification under natural conditions. 


\section{Methods}

It was not possible to record data blind because our study involved observations of focal animals bearing VIE tags.

\section{Study species}

Neolamprologus pulcher is a small cichlid endemic to Lake Tanganyika, with a lake-wide distribution (Duftner et al. 2007). It uses crevices between rocks or small caves dug out under stones for reproduction and shelter (Taborsky and Limberger 1981; Balshine et al. 2001; Heg et al. 2008). Groups of $N$. pulcher consist of a dominant breeding pair, i.e. a male and female largely monopolising reproduction (Hellmann et al. 2015), and several helpers of both sexes and various sizes (Groenewoud et al. 2016). Helpers increase the dominants' reproductive success (Taborsky 1984; Brouwer et al. 2005; Jungwirth and Taborsky 2015), and helper survival is contingent on access to refuges and the defence behaviour of larger group members (Taborsky 1984; Heg et al. 2004; Heg and Taborsky 2010). Group membership is affected by within-group aggressive interactions that are influenced by size (Dey et al. 2013), competition (Balshine et al. 2001), cooperative behaviour (Fischer et al. 2014b), and the need for help (Taborsky 1985; Zöttl et al. 2013b). Groups of N. pulcher cluster in colonies, i.e. assemblages of a few up to several hundred territories in close proximity (Heg et al. 2008; Jungwirth et al. 2015a; Groenewoud et al. 2016; Hellmann et al. 2016). Dispersal in N. pulcher typically covers only short distances (Stiver et al. 2004, 2007), and recapture rates around $50 \%$ between consecutive years are not uncommon (AJ et al. unpublished data).

The research reported here was conducted on wild individuals at a field site located at the southern tip of Lake Tanganyika (long-term field experiment), and on fish from a breeding stock population maintained at the Ethologische Station Hasli, Switzerland (the founder population of the fish used in the social integration experiment and long-term laboratory experiment originated from the same geographic area in which the field work was carried out; see Electronic Supplementary Material for additional information).

\section{Tagging procedure}

All tagging equipment (with the exception of syringes) was purchased from Northwest Marine Technology (NMT INC Northwest Marine Technology, http://www. nmt.us, PO Box 427, Ben Nevis Loop Road, Shaw Island, Washington 98286, USA), and was prepared and used following the respective user's manual (North West Marine Technology 2008).

Pilot experiments showed that recovery after tagging was quicker without anaesthesia, and all fish included in this study were consequently tagged without the use of anaesthetics. Fish tagged without anaesthetics typically resumed normal behaviour (i.e. freely swimming, feeding, interacting with conspecifics) within $5 \mathrm{~min}$ after release and thereafter showed no clear signs of discomfort (e.g. no increased scratching, hiding, swimming in unusual postures, etc.).

In short, standard $0.5 \mathrm{ml}$ insulin syringes were loaded with freshly prepared two-component VIE mixture prior to tagging. Coloured silicone was inserted by carefully moving the needle under the scales near the rear end of the desired tag's location, i.e. towards the fish's caudal fin. Upon piercing the underlying skin, the needle was moved forward to the frontal end of the desired tag position, i.e. in the direction of the fish's snout. Tags were approximately $2-4 \mathrm{~mm}$ in length and were placed as closely to the skin's surface as possible. Colour was injected as the needle was retracted, and injecting was stopped shortly before reaching the point where the skin had been initially pierced (as advised in the user's manual, see above). Each individual fish received all its tags in a single session lasting a maximum of $10 \mathrm{~min}$ between capture and release (including size measurement, tissue collection, and sex determination; see below). Tagging took place in a separate laboratory room for the social integration experiment and the long-term laboratory experiment. For the long-term field experiment, tags were applied while SCUBA diving.

Tags were placed at a total of 9 different locations on the body of $N$. pulcher (see Fig. 1), chosen to be easily distinguishable even when fish grow (Claverie and Smith 2007; Anderson et al. 2015; Sapsford et al. 2015; Schuett et al. 2017). In both long-term experiments, we investigated whether initial size differences among individual fish, and resulting differences in growth over the observation period (larger fish grew less than smaller fish: Growth $=-0.47 *$ Initial Size + 2.88; $\mathrm{t}=-11.37, p<0.01$; see Appendix 5), influenced rates of correct tag identification. The underlying assumption for this was that greater growth could increase the potential for tag misidentification. We used a total of seven different colours (black, green, orange, pink, red, white, yellow). Some of these colours do fluoresce under (near) UV light (green, orange, pink, red, yellow; North West Marine Technology 2008). However, all our experiments were carried out under ambient light, i.e. standard laboratory lighting from fluorescent lights, or the natural lighting at 10+ metres depth in Lake Tanganyika, and no fluorescence was obvious during any stage of the work. With the 9 positions and 7 colours we had at our disposal, and using 2 VIE tags per lateral side, this allowed for a total of 7056 unique individual tagging patterns (North West Marine Technology 2008). Each fish received a maximum of two tags of either the same or different colours at two different positions per lateral side. Each tag was placed on both lateral sides of an individual's body (see Fig. 1). Each fish consequently received either two or four tags in total (one or two per lateral side). Tagging of handling-control fish (h- 

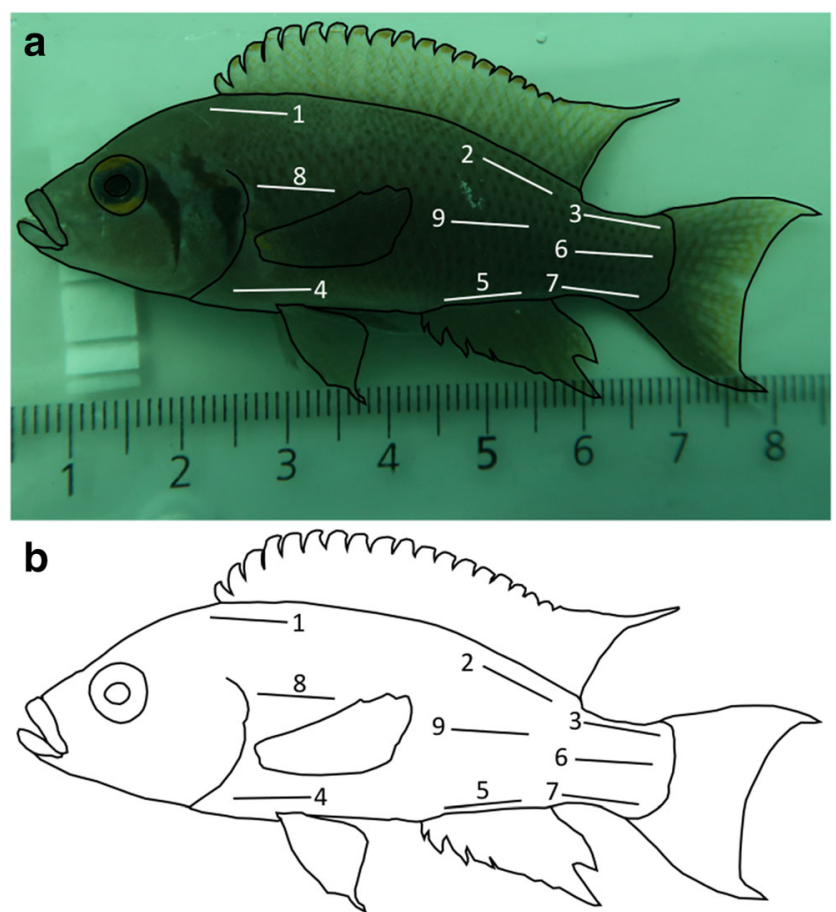

Fig. 1 Overview of the positions used for VIE tagging in N. pulcher in this study. a Photo of a $6.4 \mathrm{~cm}$ (SL) male caught in the study population (taken at approximately $12 \mathrm{~m}$ depth and using a clear plastic bag to restrain the live fish), with an outline of its body, eye, gills, and fins superimposed, as well as a schematic overview of the 9 tagging positions used (white). b The superimposed outline and schematic overview of tagging positions (black) without the original picture

control) was equivalent to actual tagging in all respects, i.e. hcontrol fish were caught and measured in the same way as all other individuals, and they were pierced with a needle for similar amounts of time and with similar injection depth as truly tagged fish. However, we used empty syringes for hcontrol fish and did not inject any fluid or gas.

\section{Social integration experiment}

In order to investigate whether VIE tags influence social dynamics in $N$. pulcher, we experimentally simulated the formation of cooperative groups while altering the presence, colour, and position of VIE tags in the most subordinate of individuals. As group membership is essential for subordinate survival and future reproduction (see above), a negative impact of VIE tags on subordinates' ability to integrate into or remain within a breeding group would render VIE tagging unsuitable for studies of these fish. A detailed description of the methods is provided in Appendix 2. In short, we allowed two large fish to form a dominant breeding pair and investigated whether a small fish would be accepted by these as a subordinate helper. In total, we gave 93 helper-sized fish $(2.1-4.1 \mathrm{~cm}$ standard length (SL); measured from the tip of the snout to the end of the caudal peduncle) the opportunity to join a pair of potential breeders (males: $n=24$, females: $n=25$ ). Standard length of breeder males varied between 6.2 and $7.8 \mathrm{~cm}$, and that of breeder females between 5.5 and $7.1 \mathrm{~cm}$. To ensure pair stability, breeder males were always larger than their respective breeder females. The potential helpers were tagged in two positions per lateral side, either near the head (positions 1 and 8, see Fig. 1) or tail (positions 3 and 7), and only one colour was used per individual (black, red, yellow, or none for h-control fish). Colours were chosen on the basis that (i) they are included in the species' normal colouration (black and yellow; Duftner et al. 2007), (ii) they are known to be important during aggressive encounters (black; Balzarini et al. 2017), or (iii) they are easy to see by human observers (red). Tagging took place a week before potential group members encountered each other for the first time. For the experiment, we first introduced the helper-sized fish to a new tank, where it was allowed to habituate. Afterwards, we introduced the breeder-sized fish. Once all three fish had been introduced to a tank, group formation was monitored for 3 days. After this time, we scored a potential helper as accepted by the prospective breeder pair if it was allowed to freely roam the pair's territory and received only low amounts of aggression (Zöttl et al. 2013b). If the potential helper was constantly attacked and/or sought shelter outside of the pair's territory, we scored it as not accepted (see Appendix 2 for additional details).

\section{Long-term laboratory experiment}

To test whether potential differences in VIE tag identification might depend on colour or position of tags, and whether VIE tags may impair individual survival, we surveyed tagged fish over the course of 1 year in the laboratory. In August 2012, we tagged 41 fish of both sexes and of various sizes $(2.9-5.9 \mathrm{~cm}$ SL) with individually unique combinations of VIE tags, and subjected 10 additional fish to the handling-control treatment. We used all 9 positions that were identified as easily distinguishable (Fig. 1) and all colours listed above (with the exception of yellow, which potentially influences intra-specific behaviour (see Results: Social integration experiment), and white, which had proven difficult to see for human observers during pilot trials). The 41 tagged fish received 130 tags in total (1-2 per lateral side per individual fish) and the $10 \mathrm{~h}$ control fish received 10 pseudo-tags (i.e. no elastomer injected; 1 per individual fish), resulting in a total of 140 individual tags to be analysed. Subsequently, these fish were transferred to two large holding tanks where they were kept in mixed-sex groups under standard laboratory conditions with 5 control fish in each tank (see Appendix for details of housing conditions, sex distribution, individual sizes, etc.). Fish were kept in these tanks for 1 year, and survival was checked daily throughout this period. In August 2013, we caught all surviving fish and recorded which of the tags we could easily and unambiguously identify, and which fish had died during the year. In addition, we recorded whether we could 
unambiguously identify the tags of any fish that died during the experimental period. Consequently, we scored a tag as 'retained' if it was clearly and unambiguously identifiable either at the end of the experiment or when the individual fish carrying it died. We again measured each individual's SL at the same time at which tag retention was determined.

\section{Long-term field experiment}

To verify that VIE tags allow for long-term tracking and recognition of individual $N$. pulcher in the wild, we surveyed tagged fish in their natural habitat. We focused our tagging efforts on one large colony at the eastern-most edge of the $N$. pulcher population at Kasakalawe Point near Mpulungu, Zambia (Balshine et al. 2001) at 10-12 m depth. East of this colony, stones are largely absent and no $N$. pulcher can be found for several hundred meters. We are confident that fish we could not recapture near the original tagging location had died rather than dispersed outside of our working range, because (i) the population of Kasakalawe Point clusters into distinct colonies interspersed by long stretches of uninhabited terrain (Heg et al. 2008; Hellmann et al. 2016), and (ii) dispersal typically occurs within rather than between colonies (Stiver et al. 2004; Dierkes et al. 2005; Stiver et al. 2007; Heg et al. 2008). The focal colony of the current study covered an area of roughly $30 \times 30 \mathrm{~m}$ (Jungwirth et al. 2015a), and was comprised of between 135 and 157 groups in a given year, several of which persisted throughout the whole observation period between September 2011 and November 2013 (Jungwirth and Taborsky 2015).

For the current study, 137 individual $N$. pulcher were caught, VIE tagged, and recaptured in various territories within the focal colony. Forty-three of these individuals were recaptured twice, i.e. they were initially caught and tagged in 2011 and subsequently recaptured in 2012 and 2013. The other 94 fish were recaptured only once in the year after initial capture. In total, of the 137 fish in this study 89 were female (mean SL at initial capture: $4.9 \mathrm{~cm}$, range 3.5-5.5) and 48 were male (mean SL at initial capture: $5.4 \mathrm{~cm}$, range 3.6-6.3).

Fish were tagged in a way similar to that described for the long-term laboratory experiment: each fish received 1-2 tags per lateral side of either the same or different colours. We predominantly used orange, pink, and red (see sample sizes in Fig. 3), because these colours proved to have the highest detectability for human observers during pilot trials. We applied tags mostly in positions 1,2 , and 9, as these were the easiest points for injection (see Figs. 1, Appendix 4.1).

A total of 263 fish of adult size (dominant males, dominant females, and large subordinates, i.e. fish approximately $3.5 \mathrm{~cm}$ SL and larger; Heg et al. 2004) were marked between September and November 2011 (160 fish) and 2012 (103 fish). All procedures (i.e. catching, measuring, determining sex, fin-clipping, and tagging) were carried out underwater, close to the respective fish's home territory, by SCUBA diving. In the following year, i.e. in 2012 and 2013, respectively, we checked for tagged fish during the same time period (see Jungwirth and Taborsky 2015 for additional details on the methods used and the colony under consideration). We observed all $N$. pulcher territories in the area for 5-10 min from close proximity $(<1 \mathrm{~m})$, using a LED underwater torch to increase visibility of tags. Any fish for which this visual examination suggested that they bore tags were subsequently caught. Upon capture, the colour and position of their tag was noted, their SL and sex were determined, and a tissue sample was taken for DNA fingerprinting (see Jungwirth et al. 2015b for details of the molecular procedures). This allowed us to verify a fish's identity independently of the respective tags, and thus to compare the observed tags with the records of actually injected tags. In addition, in 2012 and 2013, we also caught a large number of fish for which there was no indication that they had been tagged previously, subjecting them to the same measuring and sampling schedule, and tagging them (2012: $n=103 ; 2013: n=96)$. This was done to increase the number of tagged fish for recovery in future years, but also to potentially pick up fish that had completely lost their tags. We thus caught the following percentages of all adult sized fish (see above) in the colony in that respective year; 2011: 35\% (160 of 461 fish), 2012: 44\% (186 of 427 fish), and 2013: $47 \%$ (209 of 443 fish). This procedure led to the recapture of 137 previously tagged fish, representing a total of 251 tags (i.e. 1-2 tags per fish) for which we could check whether they had been identified correctly or not. We did not distinguish tag identification per lateral side of these fish.

\section{Statistical analyses}

All statistical analyses were carried out using $\mathrm{R}$ version 3.5.2 (R Development Core Team 2013), generalised linear mixed effects models (GLMMs) were fitted using the $\mathrm{R}$ package lme4 (Bates et al. 2013), and power analyses were carried out using the R package pwr (Champely et al. 2018). For all generalised linear models (GLMs) and GLMMs, statistical metrics were calculated using the 'drop1' function with $\chi^{2}$ tests ('chisq').

To analyse whether VIE tags influenced acceptance of helper-sized fish by breeders in the social integration experiment, we fitted a GLM with logit link function assuming a binomial error distribution. In this model, a helper's status (accepted or evicted) was used as the binomial response variable, the colour of the tags it received (black, red, yellow, or none), and the position at which it received its tags (head or tail, see above) were fitted as fixed effects. We also initially included the interaction between tag position and colour, but removed it as it had no significant effect $(p=0.6)$; we subsequently refitted the model without this interaction (Engqvist 
2005). Further, we included the relative size difference between the focal subordinate and the respective dominant male and female as fixed effects (absolute size difference divided by the sum of both sizes). This model had high power to detect even small effect sizes (ES; for $\alpha=0.05$ and $\mathrm{ES}=0.2$ : power $=0.92$ ).

To test whether identification of a tag in the long-term laboratory experiment was influenced by a tag's colour or position as well as the size of the fish bearing it, we performed two GLMMs with logit link function assuming a binomial error distribution. This was done to separately analyse the effects of colour and position to find the most suitable colours and positions, while ensuring that potential effects of body size on identification rates of colours and/or positions of tags were considered (the same reasoning applies to the analyses of the long-term field experiment). The first model included whether a tag's position was identified correctly (yes or no) as the binomial response variable, the tag's position (positions 1, 2, 3, 5, 6, 7, 9; see Fig. 1) and the individual fish's SL at the time of tagging were included as fixed effects, and the fish's ID and the tank in which it was kept throughout the duration of the experiment were included as random effects. This model had high power to detect even small effect sizes (for $\alpha=$ 0.05 and $\mathrm{ES}=0.2$ : power $=1$ ). The second model included whether a tag's colour was identified correctly (yes or no) as the binomial response variable, the tag's colour (black, green, orange, pink, or red) and the individual fish's SL at the time of tagging were included as fixed effects, and the fish's ID and the tank in which it was kept throughout the duration of the experiment were included as random effects. This model had high power to detect even small effect sizes (for $\alpha=0.05$ and $\mathrm{ES}=0.2$ : power $=0.98$ ). To test whether carrying VIE tags influenced a fish's survival in the long-term laboratory experiment, we performed a $\chi^{2}$ test. This test had low power to detect even large effect sizes (for $\alpha=0.05$ and ES $=0.8$ : power $=0.09$ ). To test whether initial size influenced survival in the long-term laboratory experiment, we fitted a GLM with logit link function assuming a binomial error distribution. This model included whether a fish survived the observation period (yes or no) as the binomial response variable, and the fish's initial size (SL in $\mathrm{cm}$ ) as fixed effect. This model had high power to detect even small effect sizes (for $\alpha=0.05$ and $\mathrm{ES}=$ 0.2 : power $=0.89$ ).

To test whether identification of a tag in the long-term field study was influenced by a tag's colour or position and/or by the initial size of the fish bearing it, we ran two GLMMs which included the respective binomial response variable (correct identification of position or colour: yes or no), the fixed effects of interest (tag position (positions 1, 2, 3, 5, 6, 7, 9; see Fig. 1) or colour (black, green, orange, pink, red, or white), and the individual fish's SL at the time of tagging), and two random effects (fish ID $(n=137)$ and tag ID $(n=251)$ ). Because many tags were observed in 2 years, the total sample size for this analysis was 322 ('tag observation years', i.e. number of years for which identification of single tags could be observed: 180 tags observed for a single year, 71 tags observed for 2 years: $180 * 1+71 * 2$ ). These models had high power to detect even small effect sizes (for $\alpha=0.05$ and ES = 0.2 : power $=1$ for both models). To test whether identification of a tag in the long-term field experiment was influenced by the time that had passed since its initial injection, we performed two $\chi^{2}$ tests. These tests had low power to detect small effect sizes (for $\alpha=0.05$ and $E S=0.2$ : power $=0.39$ for both models).

\section{Data availability}

The datasets analysed during the current study are available from the corresponding author on reasonable request.

\section{Results}

\section{Social integration experiment}

In total, 49 of the 93 helper-sized fish were accepted by the prospective breeders. The position at which the fish had received its tags did not influence acceptance (GLM logit link: likelihood ratio test (LRT): 0.009, $p=0.93$ ). Fish bearing yellow tags had the lowest acceptance rate (Fig. 2), but there was no statistically significant general effect of tag colour on acceptance (LRT $=5.35, p=0.15$ ). The relative size difference between the focal helper-sized fish and the respective breeders did not influence acceptance (relative size difference to dominant male: $\mathrm{LRT}=0.37, p=0.54$; to dominant female: $\mathrm{LRT}=$ $0.06, p=0.81$ ).

\section{Long-term laboratory experiment}

Of the 130 tags investigated in the long-term laboratory experiment, we were able to correctly identify the position and colour of 114 tags after 1 year. All tags that were recorded as not correctly identified were in fact completely lost. In other words, we never misinterpreted either colour or position in the laboratory study; only complete tag loss led to errors in identification. There was no detectable effect of a tag's position (GLMM logit link: LRT $=0.38, p=0.54$; Fig. Appendix 3.1) or colour (LRT $=2.3, p=0.68$; Fig. Appendix 3.2) on rates of correct tag identification after 1 year in the long-term laboratory experiment. The initial SL of the fish bearing a tag did also not influence whether its position or colour was correctly identified or not (position: LRT $=0.01, p=0.95$; colour: $\mathrm{LRT}=0.04, p=0.85$ ). Tags were recorded as lost at varying time points (2-10 months after injection) and no clear effect of colour on the timing of being recorded as lost was apparent (see Appendix 3 for details). 
Fig. 2 The acceptance of helpersized fish by prospective breeders in the social integration experiment. Black areas of bars represent the proportion of helper-sized fish accepted, white areas represent non-accepted individuals. In (a), data were sorted according to the position at which a helpersized fish had received its tags (Fig. 1; head: positions 1 and 8; tail: positions 3 and 7). In (b), data were sorted according to the colour of the tags a helper-sized fish received (no: h-control fish). Numbers above bars give the respective sample sizes of individual helper-sized fish. There was no effect of tag position on acceptance rates, but there was a weak, non-significant trend for tag colour to influence acceptance (see "Results" section) a

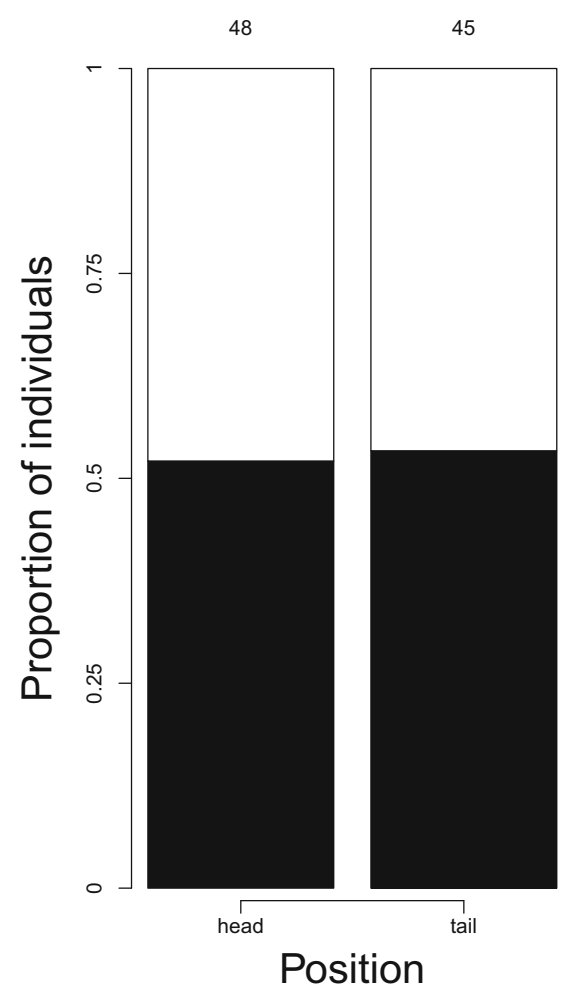

b

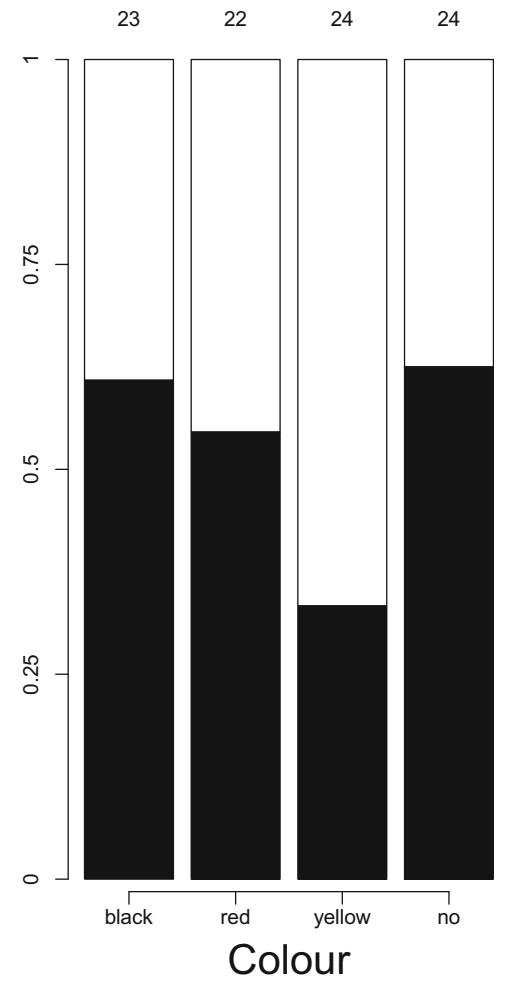

Of the 51 fish used, 7 died during the course of the experiment. All seven individuals bore tags (colours: black, orange, pink, red), i.e. none of the h-control fish died. Nevertheless, there was no significant effect indicating a potential difference in survival rates among tagged and h-control fish $\left(\chi^{2}=0.8\right.$, $p=0.37$ ). Initial size did also not influence individual survival in the long-term laboratory experiment (GLM logit link: $\mathrm{LRT}=0.27, p=0.6$; average SL of surviving and nonsurviving fish: 4.2 and $4.1 \mathrm{~cm}$, respectively).

\section{Long-term field experiment}

A total of 251 tags were considered in the long-term field experiment. Of these, 180 tags (on 92 individual fish) were checked once (i.e. approximately 1 year after their initial injection), and 71 tags (on 45 individual fish) were checked twice (i.e. approximately one and 2 years after their initial injection, respectively; the total of 'tag observation years' was thus 322, Appendix 4 and Fig. 3).

There was no effect of a tag's position on the rate of correct identification (GLMM logit link: LRT $=0.05, p=0.83$; Fig. Appendix 4.1), and the initial size of the fish bearing it did not influence whether its position was correctly identified (LRT $=$ $0.07, p=0.79$ ). However, rates of correct identification were influenced by a tag's colour in the long-term field experiment (LRT $=65.17, p<0.01$; see Fig. 3 for rates of correct identification per colour). Correct identification of a tag's colour was also influenced by the initial size of the fish bearing it (LRT $=4.68, p=0.03$ ), with tags on larger fish being identified more accurately than tags on smaller fish.

Elapsed time since tagging did not influence rates of correct identification of a tag's position in the long-term field experiment $\left(\chi^{2}=0.69, p=0.41\right)$. There was a non-significant trend

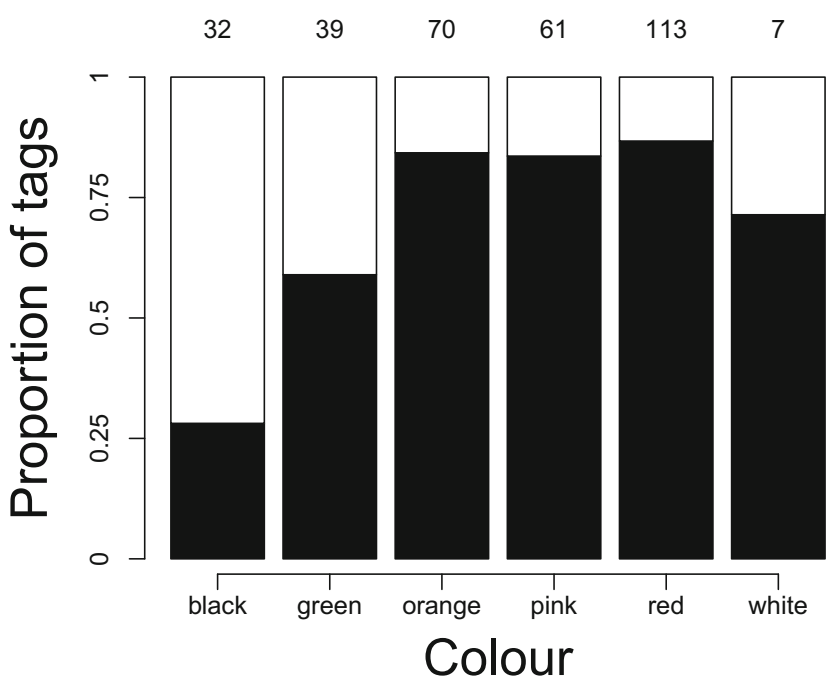

Fig. 3 Rates of correct identification of tag colour in the long-term field experiment. Black areas of bars represent the proportion of correctly identified tags, white areas represent tags that were not correctly identified. Numbers above bars give the respective sample size of individual tags. Tag colour influenced rates of correct identification (see "Results" section) 
for time since tagging to influence rates of correct identification of a tag's colour $\left(\chi^{2}=2.91, p=0.088\right)$. Unexpectedly, tags were more often correctly identified 2 years after tagging compared to identification rates in the year immediately following tagging (Fig. Appendix 4.2; 66\% correct identification in year 1 versus $\sim 80 \%$ correct identification in year 2 ).

\section{Long-term identification of individual fish}

On average, we correctly identified $78 \%$ of tags 1 year after injection (i.e. tag retained and both position and colour identified correctly; 113 of 130 tags correctly identified in the long-term laboratory experiment (86\%), and 185 of 251 tags correctly identified in the long-term field experiment (73\%); Figs. 3, Appendix 3.1, Appendix 3.2, Appendix 4.1). These data allow to estimate rates of correct recognition of individual fish based on VIE tags alone: in the absence of other means of individual identification, 31 (of 41) fish would have been recognised correctly in the long-term laboratory experiment and 86 (of 137) fish in the long-term field experiment, resulting in $75 \%$ and $63 \%$ correct individual fish recognition in either dataset.

\section{Discussion}

Colour markings are a widely used technique for tracking individuals of various taxa. To date, most studies investigating the influence and suitability of colour-tagging focus on survival and growth in either the laboratory or the field, and studies incorporating social and behavioural impacts of VIE tags are scarce (but see Frommen et al. 2015; Schuett et al. 2017; Ruberto et al. 2018 for notable exception).

Our data reveal that VIE tags represent a useful technique for conducting long-term studies in $N$. pulcher in both laboratory and field. Certain colours proved to be more useful than others; in the field, colours with a reddish hue worked particularly well (Fig. 3). The position at which a tag had been placed did not affect social interactions among fish or tag identification by human observers (Fig. 2, Appendix 3.1, Appendix 4.1), but there were differences in how easily tags could be applied to the different positions (see "Methods" section).

These findings suggest that the four caveats of VIE tagging outlined in the "Introduction" section are of little importance in our study species: rates of misidentification were low when considering only those colours and positions we identified as suitable; numbers of unique individual identifiers were sufficient for the scope of our experiments, even after exclusion of certain colours and positions; we found no strong evidence that bearing VIE tags impaired survival; and we found no strong evidence that bearing VIE tags influenced social behaviour. We will discuss each of these findings in greater detail below. Hence, if VIE tags worked as well in other species as they do in $N$. pulcher, they could be a useful tool to expand the availability of individual-based, long-term datasets from the wild also in small tropical freshwater fishes.

Nevertheless, it is important to consider also the limitations of this method (see also Matechik et al. 2013). The long-term experiments revealed the potential for tag loss or misidentifications of the position or colour of tags, which reduces the reliability of VIE tags for tracking individuals somewhat if no other identification method is used in parallel. Whether the observed error rates are acceptable will strongly depend on the questions studied. In the current study, our focus was on maximising recapture rates by choosing colours that human observers found easy to spot in the challenging lighting conditions of Lake Tanganyika. Thus, we predominantly used colours with a reddish hue, which increased detectability from afar, but reduced differentiation, leading to misidentification among orange, pink, and red. There was also a tendency for slightly higher rates of correct tag identification 2 years after tag injection compared to 1 year after injection (Fig. Appendix 4.2). On the one hand, this demonstrates that VIE tags did not deteriorate over the course of our experiment, but on the other hand, it highlights that various factors, e.g. light conditions or observer training, may introduce variation in the utility and performance of VIE tags. The fact that correct identification of a tag's colour in the long-term field experiment was higher in fish that were larger when receiving their tags (see "Results" section and Fig. Appendix 4.3) could be due to a combination of three factors: (i) reduced growth in larger fish may increase colour fidelity (see Fig. Appendix 5); (ii) larger fish may have received larger tags that are more easily identified; or (iii) larger fish may have received their tags closer to the surface of the skin, improving colour identification. While we aspired to give each individual fish a tag of roughly the same quality, i.e. similar size and depth, it is undeniable that larger individuals were easier to handle, especially underwater. Additional experiments with a focus on the effect of size on tag identification rates will be needed for further clarification.

We initially identified 9 distinct tagging positions and 7 colours as suitable for a total of over 7000 unique tagging combinations (Fig. 1; "Methods"). This would have been more than sufficient for the purposes of our long-term studies. However, in light of our findings, we decided to abandon certain positions and colours. While differences in individual growth did not affect rates of correct identification of either position or colour, other factors did: positions 4 and 8 proved to be difficult to use in the field as they are close to the gills which makes it increasingly challenging to restrain the fish in a non-invasive manner, and position 7 was too easily confused with either positions 3 or 6. Four colours were eventually avoided for various reasons (yellow: potential influence on helper acceptance; white: difficult to see for human observers; black and green: lower rates of tag identification becoming apparent throughout the field experiment). This left us with an effective count of 6 positions and 
3 colours, allowing for a total of 540 unique individual tagging patterns. If more individual identifiers are required, there are several possibilities, e.g. injecting more tags per individual fish, using additional colours, or defining more potential injection positions. Which of these measures is most suitable will depend on the needs of the respective study.

We found no evidence for lowered survival of tagged fish in the long-term laboratory experiment. However, due to the small number of h-control individuals, the power for finding weak effects was low. Additional controls could be performed in future studies to investigate other potential survival costs of tagging, e.g. injection of undyed elastomer or performing the same number of needle injections on control fish as on tagged fish. It remains unclear if, and to which extent, carrying colourful tags may increase predation risk or social aggression in the wild. Here, additional research effort is needed, e.g. using controlled predation experiments in the wild (Heg et al. 2004) or under semi-natural conditions (Bouska and Paukert 2010), or using computer simulated stimuli to test potential predator preferences for tagged or untagged fish (Fischer et al. 2014a; Balzarini et al. 2017).

A special concern in a species characterised by complex social organisation is that tag colour might influence behavioural interactions. In $N$. pulcher, the head region shows distinct yellow, blue and black marks (Duftner et al. 2007; Kohda et al. 2015; Balzarini et al. 2017). For this reason, we focused on black and yellow in the social integration experiment, adding the colour that pilot experiments had shown to be best visible to human observers (red). While no colour (or colour/ position combination) led to a significant increase or decrease of acceptance rates compared to h-control fish (Fig. 2), yellow seemed to indicate negative effects on acceptance (Fig. 2b). Hence, we decided to use a conservative approach and excluded this colour from further studies until its potential effects have been elaborated by additional scrutiny.

In conclusion, our work shows that VIE tags can be a useful tool to track individual fish in the wild over extended periods of time, which is corroborated by recent long-term studies of $N$. pulcher (Zöttl et al. 2013a; Jungwirth and Taborsky 2015; Jungwirth et al. 2015b, 2016). However, our results also highlight that VIE tagging alone may be insufficient for absolute accuracy in individual identification over longer periods of time. Depending on the study species of choice and the specific research question, it may be advisable to either use additional identifiers or to perform a separate study clarifying the reliability of this method. VIE tags in general, and certain colours in particular, may influence social behaviour (Frommen et al. 2015; Schuett et al. 2017; Ruberto et al. 2018), and the reliability of retention and individual recognition over longer time periods may be considerably lower than $100 \%$ (Coombs and Wilson 2008; Kozłowski et al. 2017; this study: long-term experiments). Thus, species-specific optimisation of the VIE-tagging procedure is advisable, with particular focus on social behaviour and the light environment in which the work is conducted.

Acknowledgments The contributions of Pierpaolo Brena, Dario Josi, Isabel Keller, and Jonas Walker were crucial for data collection in the field. Evi Zwygart maintained optimal work conditions in the lab. Danielle Bonfils performed the molecular analyses. We thank Danny Sinyinza and the staff at the Department of Fisheries in Mpulungu, Zambia, for logistic support, and the Zambian Ministry of Agriculture and Cooperatives for the study permission. We are grateful to Celestine Mwewa and the staff at the Tanganyika Science Lodge for their hospitality. The manuscript greatly benefited from insightful comments by two anonymous reviewers. We dedicate this work to our friend and colleague Hirokazu Tanaka.

Authors' contributions $\mathrm{AJ}, \mathrm{VB}, \mathrm{MZ}, \mathrm{AS}, \mathrm{MT}$ and JGF conceived the study and planned the experiments. MT and JGF organised funding. MZ established the general method of VIE tagging in the test species. AJ, VB, $\mathrm{AS}$ and JGF collected data. AJ analysed the data and wrote the first draft of the manuscript. AJ, VB, MZ, MT and JGF revised the manuscript. All authors approved the final draft of the manuscript.

Funding This research was funded by grants of the Swiss National Science Foundation (31003A_156152 to MT and 31003A 144191 to JGF).

\section{Compliance with ethical standards}

Conflict of interest The authors declare that they have no conflicts of interest.

Ethical approval All applicable international, national, and/or institutional guidelines for the care and use of animals were followed. As a member of swissuniversities (swissuniversities.ch), the University of Bern demands and promotes compliance with the conventions and principles defined by swissuniversities. The field work reported here complied with Zambian laws and was carried out in agreement with local authorities under the Memorandum of Understanding issued by the Department of Fisheries: Ministry of Agriculture and Cooperatives, Zambia, dated March 20th 2009. The laboratory work was certified under Swiss Federal Veterinary Office licence BE 74/15, in which it was specified that tagging of fish would be carried out without the use of anaesthesia.

Open Access This article is distributed under the terms of the Creative Commons Attribution 4.0 International License (http:// creativecommons.org/licenses/by/4.0/), which permits unrestricted use, distribution, and reproduction in any medium, provided you give appropriate credit to the original author(s) and the source, provide a link to the Creative Commons license, and indicate if changes were made.

\section{References}

Anderson KP, Byer NW, McGehee RJ, Richards-Dimitrie T (2015) A new system for marking hatchling turtles using Visible Implant Elastomer. Herpetol Rev 46:25-27

Balshine S, Leach B, Neat FC, Reid H, Taborsky M, Werner N (2001) Correlates of group size in a cooperatively breeding cichlid fish (Neolamprologus pulcher). Behav Ecol Sociobiol 50:134-140

Balshine-Earn S, Lotem A (1998) Individual recognition in a cooperatively breeding cichlid: evidence from video playback experiments. Behaviour 135:369-386 
Balzarini V, Taborsky M, Villa F, Frommen JG (2017) Computer animations of color markings reveal the function of visual threat signals in Neolamprologus pulcher. Curr Zool 63:45-54

Bates D, Maechler M, Bolker BM, Walker S (2013) lme4: linear mixedeffects models using Eigen and S4. R package version 1.1-7, https:// cran.r-project.org/web/packages/lme4/index.html

Bouska WW, Paukert CP (2010) Effects of visible implant elastomer mark colour on the predation of red shiners by largemouth bass. Fish Manag Ecol 17:294-296

Brouwer L, Heg D, Taborsky M (2005) Experimental evidence for helper effects in a cooperatively breeding cichlid. Behav Ecol 16:667-673

Catalano MJ, Chipps SR, Bouchard MA, Wahl DH (2001) Evaluation of injectable fluorescent tags for marking centrarchid fishes: retention rate and effects on vulnerability to predation. N Am J Fish Manag 21:911-917

Champely S, Ekstrom C, Dalgaard P, Gill J, Weibelzahl S, Anandkumar A, Ford C, Volcic R, De Rosario H (2018) R package pwr. https:// cran.r-project.org/web/packages/pwr/index.html

Claverie T, Smith IP (2007) A comparison of the effect of three common tagging methods on the survival of the galatheid Munida rugosa (Fabricius, 1775). Fish Res 86:285-288

Clutton-Brock TH, Sheldon BC (2010) Individuals and populations: the role of long-term, individual-based studies of animals in ecology and evolutionary biology. Trends Ecol Evol 25:562-573

Coombs JA, Wilson JL (2008) Use of visible implant fluorescent elastomer (VIE) tag technique on darters (Teleostei: Percidae): mortality and tag retention. Southeast Fish Council Proc 50:1-7

Croft DP, Krause J, James R (2004) Social networks in the guppy (Poecilia reticulata). Proc R Soc Lond B 271:S516-S519

Dey CJ, Reddon AR, O'Connor CM, Balshine S (2013) Network structure is related to social conflict in a cooperatively breeding fish. Anim Behav 85:395-402

Dierkes P, Heg D, Taborsky M, Skubic E, Achmann R (2005) Genetic relatedness in groups is sex-specific and declines with age of helpers in a cooperatively breeding cichlid. Ecol Lett 8:968-975

Duftner N, Sefc KM, Koblmüller S, Salzburger W, Taborsky M, Sturmbauer C (2007) Parallel evolution of facial stripe patterns in the Neolamprologus brichardi/pulcher species complex endemic to Lake Tanganyika. Mol Phylogenet Evol 45:706-715

Engqvist L (2005) The mistreatment of covariate interaction terms in linear model analyses of behavioural and evolutionary ecology studies. Anim Behav 70:967-971

Fischer S, Taborsky B, Burlaud R, Fernandez AA, Hess S, Oberhummer E, Frommen JG (2014a) Animated images as a tool to study visual communication: a case study in a cooperatively breeding cichlid. Behaviour 151:1921-1942

Fischer S, Zöttl M, Groenewoud F, Taborsky B (2014b) Group-sizedependent punishment of idle subordinates in a cooperative breeder where helpers pay to stay. Proc R Soc B 281:20140184

FitzGerald JL, Sheehan TF, Kocik JF (2004) Visibility of visual implant elastomer tags in Atlantic salmon reared for two years in marine netpens. N Am J Fish Manag 24:222-227

Frommen JG, Hanak S, Thünken T, Schmidl CA (2015) Visible Implant Elastomer tagging influences social preferences of zebrafish (Danio rerio). Behaviour 152:1765-1777

Groenewoud F, Frommen JG, Josi D, Tanaka H, Jungwirth A, Taborsky M (2016) Predation risk drives social complexity in cooperative breeders. Proc Natl Acad Sci U S A 113:4104-4109

Heg D, Taborsky M (2010) Helper response to experimentally manipulated predation risk in the cooperatively breeding cichlid Neolamprologus pulcher. PLoS One 5:e10784

Heg D, Bachar Z, Brouwer L, Taborsky M (2004) Predation risk is an ecological constraint for helper dispersal in a cooperatively breeding cichlid. Proc R Soc Lond B 271:2367-2374
Heg D, Heg-Bachar Z, Brouwer L, Taborsky M (2008) Experimentally induced helper dispersal in colonially breeding cooperative cichlids. Environ Biol Fish 83:191-206

Hellmann JK, Ligocki IY, O'Connor CM, Reddon AR, Garvy KA, Marsh-Rollo SE, Gibbs HL, Balshine S, Hamilton IM (2015) Reproductive sharing in relation to group and colony-level attributes in a cooperative breeding fish. Proc R Soc B 282:20150954

Hellmann JK, Sovic MG, Gibbs HL, Reddon AR, O'Connor CM, Ligocki IY, Marsh-Rollo S, Balshine S, Hamilton IY (2016) Within-group relatedness is correlated with colony-level social structure and reproductive sharing in a social fish. Mol Ecol 25: 4001-4013

Henrich T, Hafer N, Mobley KB (2014) Effects of VIE tagging and partial tissue sampling on the immune response of three-spined stickleback Gasterosteus aculeatus. J Fish Biol 85:965-971

Jungwirth A, Taborsky M (2015) First- and second-order sociality determine survival and reproduction in cooperative cichlids. Proc R Soc B 282:20151971

Jungwirth A, Josi D, Walker J, Taborsky M (2015a) Benefits of coloniality: communal defence saves anti-predator effort in cooperative breeders. Funct Ecol 29:1218-1224

Jungwirth A, Walker J, Taborsky M (2015b) Prospecting precedes dispersal and increases survival chances in cooperatively breeding cichlids. Anim Behav 106:107-114

Jungwirth A, Brena PF, Keller I, Taborsky M (2016) Polygyny affects paternal care, but not survival, pair stability, and group tenure in a cooperative cichlid. Behav Ecol 27:593-600

Koenig WD, Dickinson JL (eds) (2016) Cooperative breeding in vertebrates: studies of ecology, evolution, and behavior. Cambridge University Press, Cambridge

Kohda M, Jordan LA, Hotta T, Kosaka N, Karino K, Tanaka H, Taniyama M, Takeyama T (2015) Facial recognition in a group-living cichlid fish. PLoS One 10:e142552

Kozłowski M, Szczepkowski M, Wunderlich K, Piotrowska I, Szczepkowska B (2017) Effect of Visible Implant Elastomers on the growth, survival and tag retention in juvenile Atlantic sturgeon (Acipenser oxyrinchus) in laboratory conditions. Aquac Res 48: 1849-1855

Malone JC, Forrester GE, Steele MA (1999) Effects of subcutaneous microtags on the growth, survival, and vulnerability to predation of small reef fishes. J Exp Mar Biol Ecol 237:243-253

Matechik CV, Mickle A, Stallings CD (2013) Experimental test of two marking methods on survival, growth, mark retention and readability on young-of-year pinfish (Lagodon rhomboides). J Exp Mar Biol Ecol 440:49-53

Moffatt C (2013) Using Visible Implant Elastomer to tag insects across life stages: a preliminary investigation with blow flies (Diptera: Calliphoridae). Can Entomol 145:466-470

Murray DL, Fuller MR (2000) A critical review of the effects of marking on the biology of vertebrates. In: Boitani L, Fuller TK (eds) Research techniques in animal ecology: controversies and consequences. Columbia University Press, New York, pp 15-64

Neenan STV, Hodgson DJ, Tregenza T, Boothroyd D, Ellis CD (2015) The suitability of VIE tags to assess stock enhancement success in juvenile European lobsters (Homarus gammarus). Aquac Res 46: 2913-2923

North West Marine Technology (2008) Visible implant elastomer tag: project manual. Northwest Marine Technology Inc, Shaw Island

Nussey DH, Froy H, Lemaitre J-F, Gaillard J-M, Austad SN (2013) Senescence in natural populations of animals: widespread evidence and its implications for bio-gerontology. Ageing Res Rev 12:214 225

Peterson D, Trantham RB, Trantham TG, Caldwell CA (2018) Tagging effects of passive integrated transponder and visual implant elastomer on the small-bodied white sands pupfish (Cyprinodon tularosa). Fish Res 198:203-208 
R Development Core Team (2013) R: a language and environment for statistical computing. R Foundation for Statistical Computing, Vienna https://www.r-project.org/

Roberts JH, Kilpatrick JM (2004) Predator feeding preferences for a benthic stream fish: effects of visible injected marks. J Freshw Ecol 19:531-538

Ruberto T, Clément RJG, Spinello C, Neri D, Macrì S, Porfiri M (2018) The tagging procedure of visible implant elastomers influences zebrafish individual and social behavior. Zebrafish 15:433-444

Sapsford S, Alford R, Schwarzkopf L (2015) Visible Implant Elastomer as a viable marking technique for common mistfrogs (Litoria rheocola). Herpetologica 71:96-101

Schuett W, Nava TF, Rahmlow N, Scherer U (2017) Artificial Visible Implant Elastomer (VIE) tags of different colour and symmetry do not influence mate choice in a cichlid. Behaviour 154:387-402

Stiver KA, Dierkes P, Taborsky M, Balshine S (2004) Dispersal patterns and status change in a co-operatively breeding cichlid Neolamprologus pulcher: evidence from microsatellite analyses and behavioural observations. J Fish Biol 65:91-105

Stiver KA, Desjardins JK, Fitzpatrick J, Neff B, Quinn S, Balshine S (2007) Evidence for size and sex-specific dispersal in a cooperatively breeding cichlid fish. Mol Ecol 16:2974-2984

Taborsky M (1984) Broodcare helpers in the cichlid fish Lamprologus brichardi: their costs and benefits. Anim Behav 32:1236-1252
Taborsky M (1985) Breeder-helper conflict in a cichlid fish with broodcare helpers: an experimental analysis. Behaviour 95:45-75

Taborsky M (2016) Cichlid fishes: a model for the integrative study of social behavior. In: Koenig WD, Dickinson JL (eds) Cooperative breeding in vertebrates: studies of ecology, evolution and behavior. Cambridge University Press, Cambridge, pp 272-293

Taborsky M, Limberger D (1981) Helpers in fish. Behav Ecol Sociobiol 8:143-145

Wong MYL, Balshine S (2011) The evolution of cooperative breeding in the African cichlid fish, Neolamprologus pulcher. Biol Rev 86:511-530

Zöttl M, Chapuis L, Freiburghaus M, Taborsky M (2013a) Strategic reduction of help before dispersal in a cooperative breeder. Biol Lett 9: 20120878

Zöttl M, Frommen JG, Taborsky M (2013b) Group size adjustment to ecological demand in a cooperative breeder. Proc R Soc B 280: 20122772

Publisher's note Springer Nature remains neutral with regard to jurisdictional claims in published maps and institutional affiliations. 